\title{
Thermal distribution analysis of inner defects in TSV
}

\author{
Chuan Kai Jiang ${ }^{1}$, Lei $\mathrm{Nie}^{1}$, Wen Jia, and Yu Ning Zhong \\ ${ }^{1}$ School of Mechanical Engineering, Hubei University of Technology, Wuhan, China
}

\begin{abstract}
In order to uncover the external manifestations of TSV internal defects, the finite element models of typical internal defects, which were filling missing, axial cavity and end cavity, were established. The thermal analysis was carried out using thermoelectric coupling method. The temperature distribution of TSV with and without defects were obtained. And the temperature variation profiles on the defined paths of TSV layer were also analyzed. The analysis indicated that all the defective TSV showed distinct temperature distribution with the defect-free TSV. Among three typical defects, TSV with filling missing showed the most obvious difference on the temperature distribution and path variation. TSV with end cavity has relatively weak affect and the slightest defect was TSV with axial cavity. Therefore, it could be seen that the external temperature difference caused by the internal defects of TSV could provide effective information for the identification and detection in TSV with internal defects.
\end{abstract}

\section{Introduction}

In the process of photoelectric manufacturing, microelectronic packaging was one of the most critical and difficult steps, which determined the electrical and mechanical properties of the device. In order to meet the requirements of modern IC product packaging, 3D packaging technology was presented. Among current 3D packaging technologies, TSV (Through Silicon Via) had attracted extensive attention due to its unique technological characteristics. Because the interconnect channel passed directly through the wafer (or chip), it not only improved the device integration, but also reduced the interconnection delay and raised the speed of the device ${ }^{[1]}$. However, because of the development trend of small aperture, high density and aspect ratio, the defects, such as filling missing, end cavity and axial cavity, were easily found which would lead to serious reliability problems. While most of these defects were located within the inner part of device package, conventional methods were hardly able to detect them directly. Thus, the inner defect inspection was becoming a research focus. In 2016, Shen J extracted seven features representative of TSVs from the images, and then inputted them into a self-organizing map (SOM) network for classification and testing. The results demonstrated that the normal TSVs and defective TSVs could be distinguished obviously by SOM network ${ }^{[2]}$. In 2016, Li F established a numerical model of $\mathrm{Cu}$-filled TSV and analyzed the effect of defects for TSV thermal mechanical stress. The results showed that thermal stress distribution in TSV was distinct with the different shape and location of defect ${ }^{[3]}$. In 2017, in order to optimize TSV design and the quality of via filling, Pan Y established a numerical model of $\mathrm{Cu}$-filled TSV to simulate and analyze the effect of diameter, aspect ratio (AR) and defects on TSV thermal stress and deformation. Simulation results showed that the equivalent stress and total deformation of TSV increased as the increase of the diameter of TSV. The effect of aspect ratio on equivalent stress was very little ${ }^{[4]}$. These researchers investigated the influence of some characteristic parameters of TSV on the temperature distribution, but did not solve the problem of some information about TSV with inner defects.

For TSV 3D-packaging, the TSV with internal defects showed abnormal temperature distribution under the electric-thermal composite load at work. If the corresponding temperature signal could be captured, the internal defects of TSV could be identified to realize the active online detection of internal defects. This paper was based on thermoelectric coupling method. And the temperature distribution inside the TSV work had been carried on the simulation analysis, which fully revealed the trend of the internal temperature distribution with different defects and provided a theoretical basis for discovery and orientation of internal defects in TSV.

\section{Principle of thermoelectric coupling analysis}

The temperature distribution of the chip module was an external result of the direct coupling of two physical fields, heat and electricity, which had a high degree of nonlinearity.

The general expression of the finite element of the heat conduction equation was as follows:

\footnotetext{
* Corresponding author: 1jiangchuankai123@163.com
} 


$$
\begin{aligned}
& C^{T} \dot{T}+K^{T}=Q \\
& Q=Q^{n d}+Q^{\mathrm{c}}+Q^{g}+Q^{j}
\end{aligned}
$$

The general expression of the finite element of the electric conduction equation was as follows:

$$
K^{V} \bullet V=I^{n d}
$$

Among, $C^{T}$ was specific heat matrix, $K^{T}$ was heat conduction matrix, $K^{V}$ was conductivity matrix, $T$ was temperature coefficient of node, $\dot{T}$ was temperature vector that changed with time on a node, $V$ was node voltage vector, $Q^{\text {nd }}$ was thermal flow vector on the node, Ind was current vector, which was applied on a node, $Q^{c}$ was surface thermal convection vector, $Q^{g}$ was external heat flow vector, $Q^{j}$ was heat generation rate vector of Joule heat of internal heat source.

Backward difference was used in time, there was the following:

$$
\left(\frac{1}{\Delta t} C^{T}+K^{T}\right) T_{n}=Q+\frac{1}{\Delta t} C T T_{n-1}
$$

This formula was used to calculate the temperature of the model node at each time $\Delta t$.

When chip module was working, the temperature of the device increased due to the working current. Along with the temperature rising, the material properties, such as the resistance, thermal conductivity and specific heat, were changed correspondingly, resulting in the thermalelectric coupling effect. The internal heat source vector $Q^{j}$ was calculated by the following form:

$$
Q^{j}=\int_{v o l}\{N\} V_{g} \sigma d(v o l)
$$

Among, $N$ was element shape function, $V_{g}$ was voltage gradient vector, $\sigma$ was conductivity matrix. The formula for calculating the voltage gradient vector was as follows:

$$
V_{g}=B V_{n-1}
$$

Among, $B$ was the matrix of the shape function $x, y, z$ to the direction differential. $V_{g-1}$ was node last iteration voltage. Because of the direct coupling between the heat conduction equation and the electric conduction equation, the solution of the whole equation group was accomplished through iteration.

\section{Modeling}

\subsection{TSV chip interconnection structure model}

The cross section of 3D-TSV stack chip packaging structure was shown in Fig. 1. In Fig. 2, the top layer was the chip, the second layer and the fourth layer were the welding ball and resin filling material between the chips (solder layer), the third layer was the pillars and the Si filling layer, and the substrate layer was the bimaleimide resin substrate. The schematic diagram of finite element mesh was shown in Fig. 3. The feature sizes of the model were respectively chip, TSV pillars, solder, substrate, and TSV layer. And the model dimension parameters were shown in Table 1. The simulation parameters (density, resistivity and thermal conductivity) used in the finite element analysis were shown in Table 2.

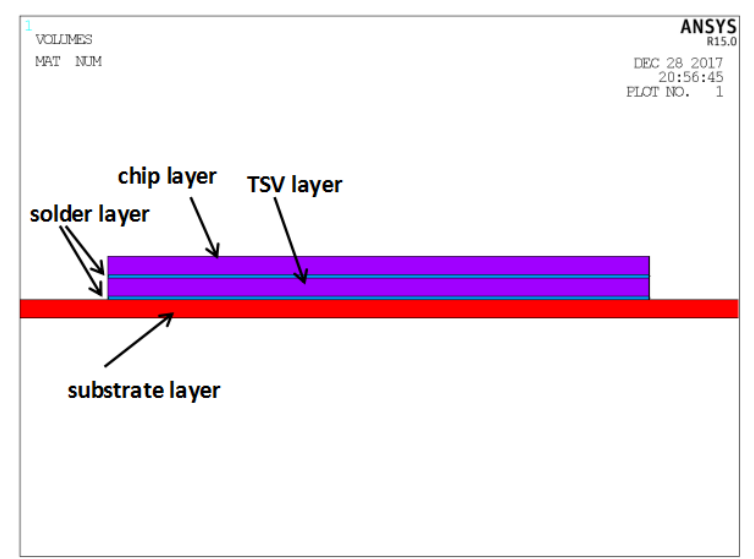

Fig. 1.The schematic diagram of the cross-section structure

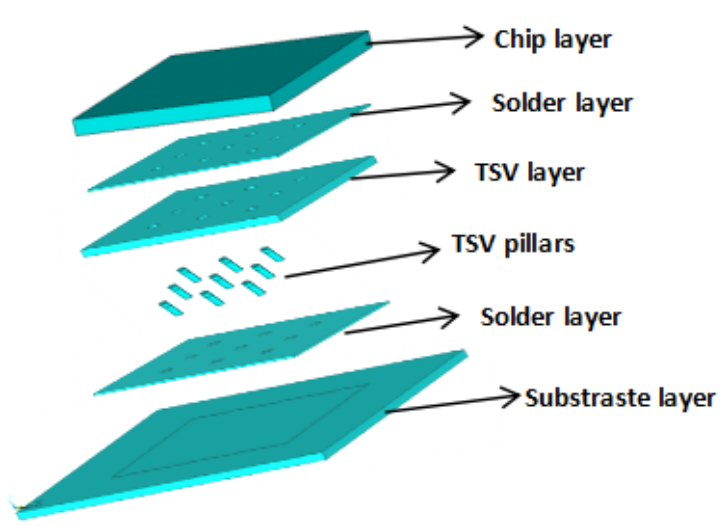

Fig. 2. Structure of TSV structure

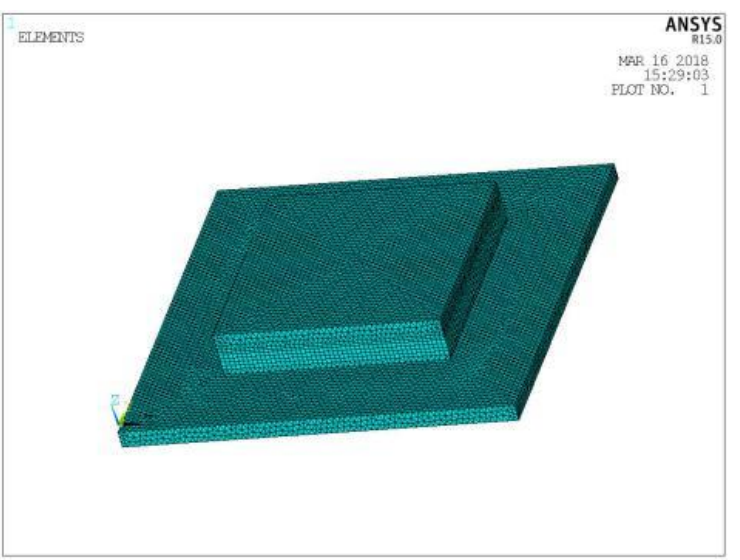

Fig. 3. Finite element mesh its overall structure

Table 1. Feature size of each module

\begin{tabular}{ccccc}
\hline & $\begin{array}{c}\text { Lengt } \\
\mathrm{h} \\
(\mathrm{mm})\end{array}$ & $\begin{array}{c}\text { Widt } \\
\mathrm{h} \\
(\mathrm{mm})\end{array}$ & $\begin{array}{c}\text { High } \\
(\mu \mathrm{m})\end{array}$ & $\begin{array}{c}\text { Radius } \\
(\mu \mathrm{m})\end{array}$ \\
\hline 1.Copper & --- & --- & 300 & 100 \\
cylinder & & 4 & 300 & --- \\
2.Silicon chip & 4 & 7 & 300 & --- \\
3.Substrate & 7 & 7 & 100 & -- \\
4.Solder & 4 & 4 & 300 & --- \\
5.TSV layer & 4 & 4 & & \\
\hline
\end{tabular}


Table 2. Simulation parameters

\begin{tabular}{ccccc}
\hline $\begin{array}{c}\text { Thermal } \\
\text { conductivity } \\
{[\mathrm{W} /(\mathrm{m} \cdot \mathrm{k})]}\end{array}$ & $\begin{array}{c}\text { Electrical } \\
\text { resistivity } \\
(\Omega \cdot \mathrm{m})\end{array}$ & $\begin{array}{c}\text { Specific } \\
\text { heat } \\
\text { capacity } \\
{[\mathrm{J}}\end{array}$ & $\begin{array}{c}\text { Density } \\
\left(\mathrm{kg} / \mathrm{m}^{3}\right)\end{array}$ \\
\hline 1 & 390 & $1.75 \mathrm{e}-8$ & 385 & 8950 \\
2 & 124 & 1 & 700 & 2330 \\
3 & 0.3 & 0.1 & --- & 1550 \\
4 & 57 & 0.1 & --- & 8740 \\
5 & 124 & 0.1 & 700 & 2330 \\
\hline
\end{tabular}

\subsection{Common defect types}

In the process of TSV manufacturing, three types of inner defects in TSV usually arose, which were shown in Fig. 4, as (a) filling missing (radius $100 \mu \mathrm{m}$ ), (b) axial cavity (radius $80 \mu \mathrm{m}$ ) and (c) end cavity (radius $80 \mu \mathrm{m}$, height $100 \mu \mathrm{m}$ ). All the defects located in the same pillar as indicated in Fig. 5.

In order to investigate the influence of different defects and pillars self-heating on the temperature distribution, thermoelectric coupling method was used in TSV structure thermal analysis. Thus, the solid 226 thermal unit was selected. And the unit was high order form with including 20 nodes, which had 5 degrees of freedom. It could improve the analysis precision and was often used in thermoelectric coupling analysis. The grid division adopted the sweep method directly. The grid contained 178948 grid cells in Fig. 3. The same load was respectively applied to the TSV pillars with different defects on the chip module. The zero potential was applied to the bottom surface of the 9 TSV pillars. And the voltage of the $1.5 \mathrm{~V}$ was applied to the upper surface of the chip layer. The boundary conditions of all models were following: the ambient temperature was $20^{\circ} \mathrm{C}$. And the surface of each part was convective heat transfer with the air. The convective heat transfer coefficient was $15 \mathrm{~W} /\left(\mathrm{m}^{2} \cdot{ }^{\circ} \mathrm{C}\right)$. And the corresponding material properties were applied to different materials.

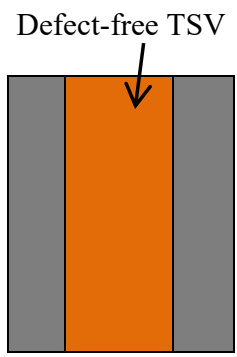

(b)Axial cavity

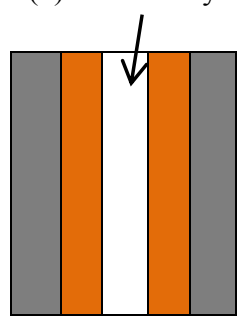

(a)Filling missing

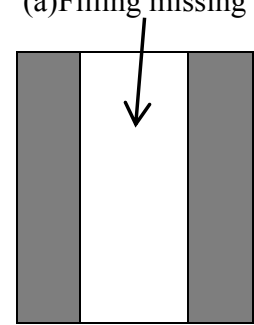

(c)End cavity

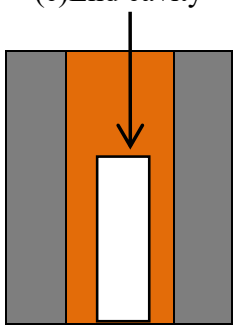

Fig. 4. Common defect profiles of TSV interconnect structure

\section{Simulation results and analysis}

The finite element analysis models of the TSV with defects were established, according to the above three kinds of inner defect types in TSV. The corresponding load and boundary conditions were applied. The nephogram results of the TSV with inner defects were obtained. The temperature distribution of the key layers was also given and compared with that of TSV without defects.

\subsection{Temperature distribution of TSV layer under various defects}

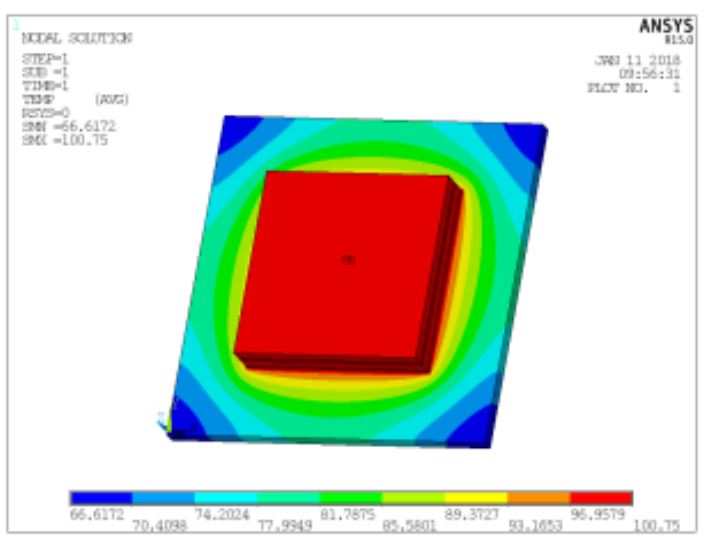

Fig. 5. Temperature distribution of TSV with defect-free

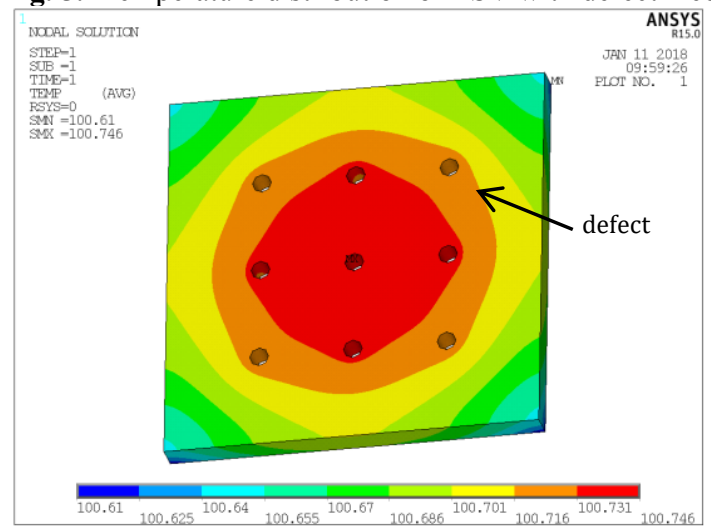

(a) Temperature distribution of the chip layer without defects

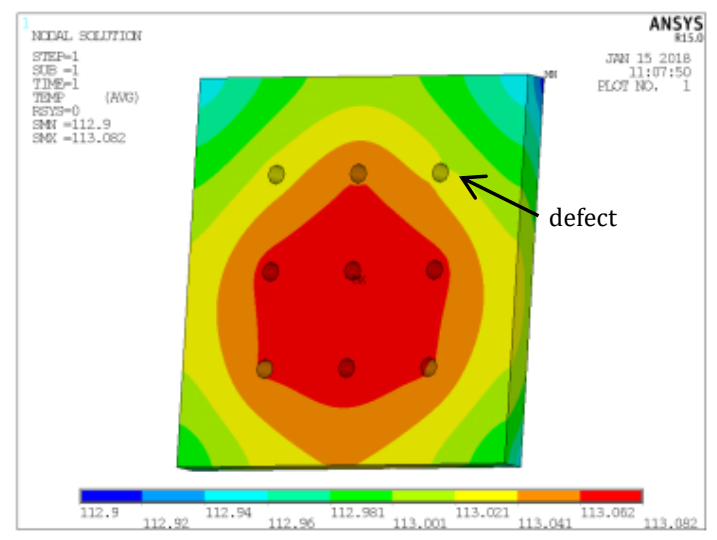

(b) Temperature distribution of the chip layers with filling missing 


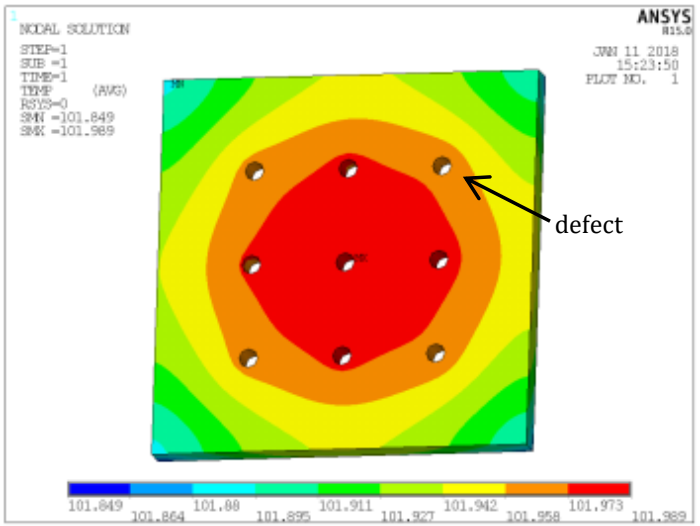

(c) The temperature distribution of the chip layer under the axial cavity

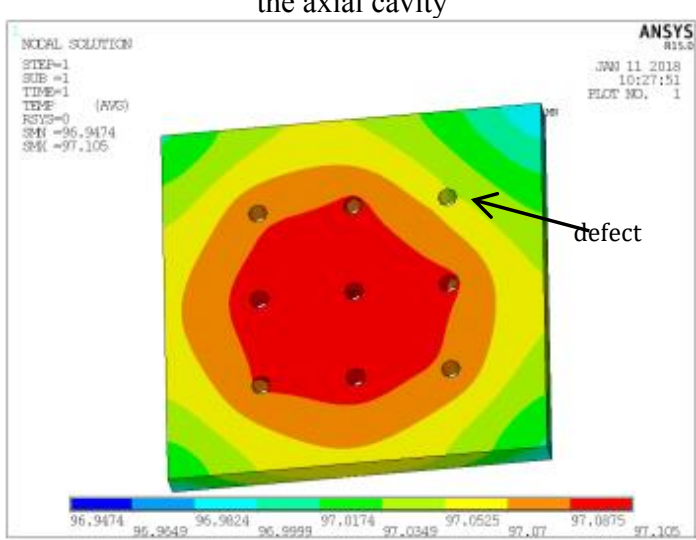

(d) Temperature distribution of chip layer under the end cavity

Fig..6. Temperature distribution of TSV layer under defects

In Fig. 5, the overall temperature distribution of defect-free TSV was given. The surface of the distribution nephogram was radial. It was easily found that the heat gradually transmitted from the center to the edges. The highest and lowest temperature were $100.75^{\circ} \mathrm{C}$ and $66.62^{\circ} \mathrm{C}$ respectively. The temperature distribution of TSV layer under various defects was shown in Fig. 6. The TSV layers of all three kinds of defects had obvious differences compared with that of defect-free TSV. Furthermore, as seen from Fig. 5 and Fig. 6, the peak temperature of TSV with filling missing, end cavity and axial cavity were $113.082^{\circ} \mathrm{C}, 101.99^{\circ} \mathrm{C}$ and $97.105^{\circ} \mathrm{C}$ respectively. That was to say, when compared to defect-free one $\left(100.732^{\circ} \mathrm{C}\right)$, TSV with filling missing defect led to the highest temperature difference $\left(12.332^{\circ} \mathrm{C}\right)$ as shown in Fig. $6(\mathrm{~b})$. The second highest difference was $3.645^{\circ} \mathrm{C}$ caused by the end cavity defect as shown in Fig. 6(d). And the lowest difference due to the axial cavity was $1.239^{\circ} \mathrm{C}$ in Fig. 6(c).

\subsection{Temperature profile in observing path}

As shown in Fig. 7, an observation path of was defined from point $A$ to $B$ on the substrate to investigate the node temperature variation. In comparison with the node temperature profile of the defect-free TSV in Fig. 8, the temperature variation profile of TSV with filling missing had the most obvious different, and that of axial cavity was the most inconspicuous. Another observation path was defined from point $\mathrm{C}$ to point $\mathrm{D}$ on the TSV layer in Fig. 9. The path temperature variation profiles showed in Fig. 10. T0 was the node temperature at $\mathrm{C}$ point and had different values for defect-free, filling missing, axial cavity and end cavity defect in order to facilitate the comparison, which were $100.61^{\circ} \mathrm{C}, 112.94^{\circ} \mathrm{C}, 101.85^{\circ} \mathrm{C}$ and $96.982^{\circ} \mathrm{C}$ respectively. It was obvious that the temperature profiles of various defects showed different patterns.

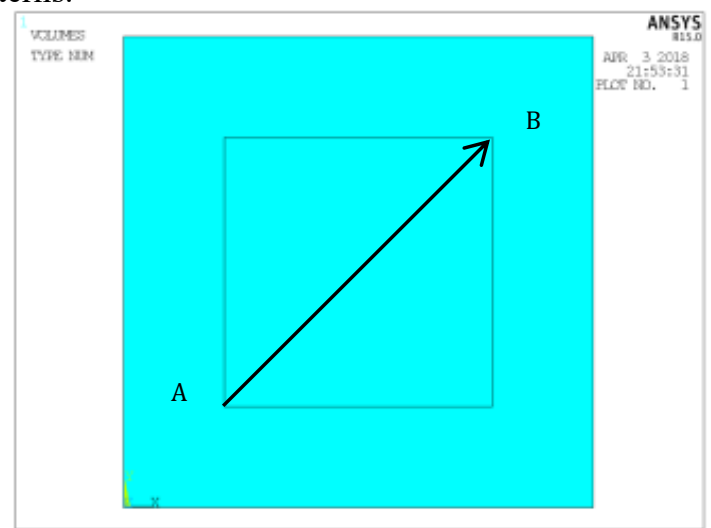

Fig. 7. A schematic diagram of the observation path on the substrate layer

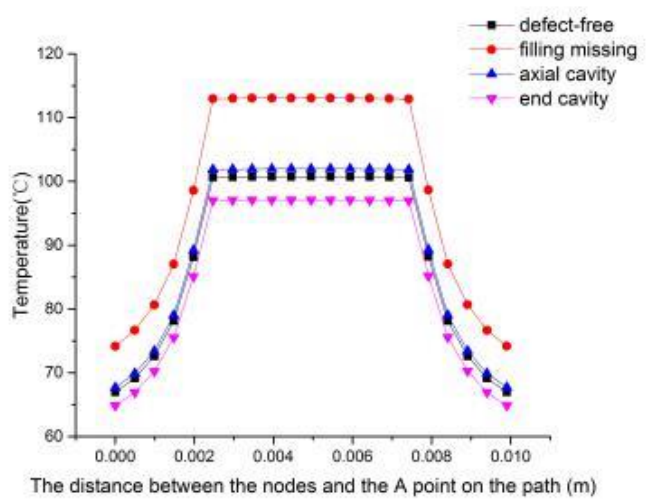

Fig. 8. Temperature profiles with various defects on the substrate layer

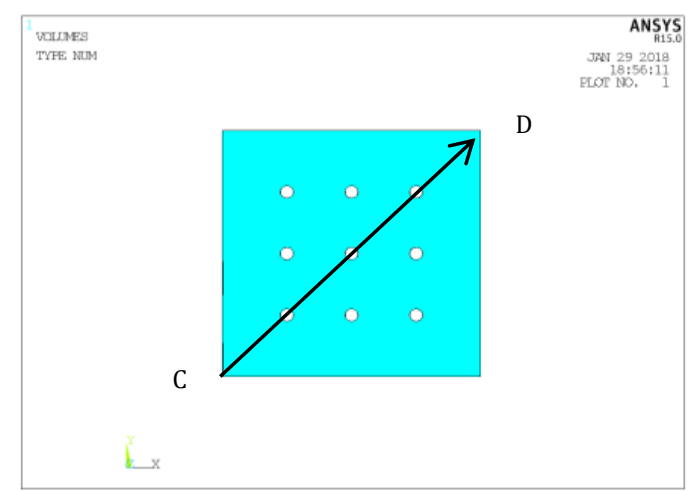

Fig. 9. A schematic diagram of the observation path on the TSV layer 


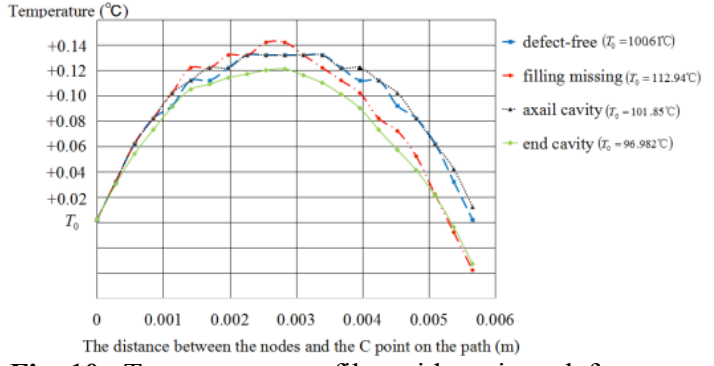

Fig. 10. Temperature profiles with various defects on the TSV layer

\section{Conclusion}

The finite element models of TSV packaging with three typical defects were established. These models were analyzed using thermoelectric coupling method and compared with the TSV without defect under the same condition. The corresponding temperature distribution and variation profiles were obtained. All kinds of defects showed unique temperature patterns which were obviously different from the defect-free TSV. Among these defects, filling missing had the most evident effect on temperature pattern. The end cavity defect took the second place and axial cavity was the weakest one. According to the analysis result, different defects obeyed different temperature distribution rules, which could be used to monitor the temperature of TSV packaging and realize the detection and positioning of TSV internal defects.

\section{Acknowledgments}

This work was funded by the open Foundation of National Key Laboratory of Precision Testing Techniques and Instrument (pilab1708), Key Project of Educational Commission of Hubei Province of China
(D20131407), and Special Fund in the Public interest of General Administration of Quality Supervision Inspection and Quarantine of P.R.China (201310004).

\section{References}

1. Zhao Z, Tong Z. 3D-TSVthe continuation of Moore's law effective way of $[\mathrm{J}]$. Electronic industry special equipment,2011,40(03):10-16.

2. Shen J, Chen P, Su L, et al. X-ray inspection of TSV defects with self-organizing map network and Otsu algorithm[J]. Microelectronics Reliability, 2016, 67:págs. 129-134.

3. Li F, Xiao C, He H, et al. Investigation on the defect induced thermal mechanical stress for TSV[C]// International Conference on Electronic Packaging Technology. IEEE, 2016:713-715.

4. Pan Y, Li F, He H, et al. Effects of dimension parameters and defect on TSV thermal behavior for 3D IC packaging[J]. Microelectronics Reliability, 2017, 70:97-102.

5. Yuan Y. Thermodynamic analysis of the three dimensional package of silicon through hole [D]. Shanghai Jiao Tong University, 2013.

6. Li C, Xie X, Shang Y, Huang H, Zou M. Transmission performance analysis of TSV interconnect structure based on electrothermal coupling effect $[\mathrm{J}]$. modern electronic technology, 2017,40 (08): 4-7.

7. Huang Q, Shang Y, Zhang M, Li C, Yue K. TSV thermal structure coupling analysis $[\mathrm{J}]$. Electronic components and materials, 2015,34 (04): 74-78.

8. Wang H. TSV modeling, heat transfer and loading analysis for three-dimensional laminated package [D]. Xi'an Electronic and Science University, 2012. 